\title{
77.
}

\section{ON THE ORDER OF CERTAIN SYSTEMS OF ALGEBRAICAL EQUATIONS.}

[From the Cambridge and Dublin Mathematical Journal, vol. Iv. (1849), pp. 132-137.(1)]

Suppose the variables $x, y \ldots$ so connected that any one of the ratios $x: y: z, \ldots$ or, more generally, any determinate function of these ratios, depends on an equation of the $\mu^{\text {th }}$ order. The variables $x, y, z \ldots$ are said to form a system of the $\mu^{\text {th }}$ order.

In the case of two variables $x, y$, supposing that these are connected by an equation $U=0$ ( $U$ being a homogeneous function of the order $\mu$ ) the variables form a system of the $\mu^{\text {th }}$ order; and, conversely, whenever the variables form a system of the $\mu^{\text {th }}$ order, they are connected by an equation of the above form.

In the case of a greater number of variables, the question is one of much greater difficulty. Thus with three variables $x, y, z$; if $\mu$ be resolvable into the factors $\mu^{\prime}, \mu^{\prime \prime}$, then, supposing the variables to be connected by the equations $U=0, V=0, U$ and $V$ being homogeneous functions of the orders $\mu^{\prime}, \mu^{\prime \prime}$, respectively, they will it is true form a system of the $\mu^{\text {th }}$ order, but the converse proposition does not hold: for instance, if $\mu$ is a prime number, the only mode of forming a system of the $\mu^{\text {th }}$ order would on the above principle be to assume $\mu^{\prime}=\mu, \mu^{\prime \prime}=1$, that is to suppose the variables connected by an equation of the $\mu^{\text {th }}$ order and a linear equation; but this is far from being the most general method of obtaining such a system. In fact, systems not belonging to the class in question may be obtained by the introduction of subsidiary

1 This memoir was intended to appear at the same time with Mr Salmon's "Note on a Result of Elimination," (Journal, vol. III. p. 169) with which it is very much connected.

C. 
variables to be eliminated: the simplest example is the following: suppose $a, b, a^{\prime}, b^{\prime}, a^{\prime \prime}, b^{\prime \prime}$ to be linear functions (without constant terms) of $x, y, z$, and write

$$
\left\{\begin{array}{l}
a \xi+b \eta=0, \\
a^{\prime} \xi+b^{\prime} \eta=0 \\
a^{\prime \prime} \xi+b^{\prime \prime} \eta=0
\end{array}\right.
$$

equations from which, by the elimination of $\xi$, $\eta$, two relations may be obtained between the variables $x, y, z$.

Suppose, however, from these three equations $x, y, z$ are first eliminated: the ratio $\xi: \eta$ will evidently be determined by a cubic equation; and assuming $\xi: \eta$ to be equal to one of the roots of this, any two of the three equations may be considered as implying the third; and will likewise determine linearly the ratios $x: y: z$. Hence any determinate function of these ratios depends on a cubic equation only, or the system is one of the third order. But the order of the system may be obtained by means of the equations resulting from the elimination of $\xi, \eta$; and since this will explain the following more general example (in which the corresponding process is the only one which readily offers itself), it will be convenient to deduce the preceding result in this manner. Thus, performing the elimination, we have

$$
L=\left(a^{\prime} b^{\prime \prime}-a^{\prime \prime} b^{\prime}\right)=0, \quad L^{\prime}=\left(a^{\prime \prime} b-a b^{\prime \prime}\right)=0, \quad L^{\prime \prime}=\left(a b^{\prime}-a^{\prime} b\right)=0 .
$$

Here the equations $L=0, L^{\prime}=0, L^{\prime \prime}=0$, are each of them of the second order, and any two of them may be considered as implying the third. For we have identically,

$$
a L+a^{\prime} L^{\prime}+a^{\prime \prime} L^{\prime \prime}=0,\left({ }^{1}\right)
$$

so that $L=0, L^{\prime}=0$, gives $a^{\prime \prime} L^{\prime \prime}=0$, or $L^{\prime \prime}=0$. Nevertheless the system is imperfectly represented by means of two equations only. For instance, $L=0, L^{\prime}=0$ do, of themselves, represent a system which is really of the fourth order. In fact, these equations are satisfied by $a^{\prime \prime}=0, b^{\prime \prime}=0$, (which is to be considered as forming a system of the first order), but these values do not satisfy the remaining equation $L^{\prime \prime}=0$. In other words, the equations $L=0, L^{\prime}=0$ contain an extraneous system of the first order, and which is seen to be extraneous by means of the last equation $L^{\prime \prime}$ : the system required is the system of the third order which is common to the three equations $L=0$, $L^{\prime}=0, L^{\prime \prime}=0$.

Suppose, more generally, that $x, y, z$ are connected by $\overline{p+1}$ equations, involving $p$ variables $\xi, \eta, \zeta \ldots$,

$$
\begin{aligned}
& a \xi+b \eta+c \zeta+\ldots=0 \\
& a^{\prime} \xi+b^{\prime} \eta+c^{\prime} \zeta+\ldots=0
\end{aligned}
$$

${ }^{1}$ Also $b L+b^{\prime} L^{\prime}+b^{\prime \prime} L^{\prime \prime}=0$ : but since by the elimination of $L^{\prime \prime}$, taking into aceount the actual values of $L$ and $L^{\prime}$, we obtain an identical equation, these two relations may be considered as equivalent to a single one. 
or what comes to the same by the equations (equivalent to two independent relations)

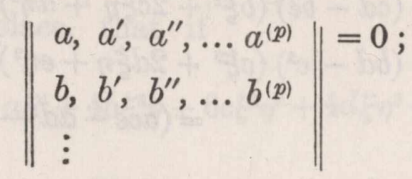

(where the number of horizontal rows is $p$ ). Consider $x, y, z$, as connected by the two equations

$$
\left|\begin{array}{lll}
a, \ldots & a^{(p-2)}, & a^{(p-1)} \\
b, \ldots & b^{(p-2)}, & b^{(p-1)} \\
\vdots &
\end{array}\right|=0, \quad\left|\begin{array}{l}
a, \ldots a^{(p-2)}, a^{p} \\
b, \ldots . b^{(p-2)}, b^{p} \\
\vdots
\end{array}\right|=0:
$$

these form a system of the order $p^{2}$, but they involve the extraneous system

$$
\left\|\begin{array}{lll}
a, & b, & \cdots \cdots \\
\vdots & & \\
a^{(p-2)}, b^{(p-2)}, \ldots
\end{array}\right\|=0 .
$$

Suppose $\phi(p)$ is the order of the system in question, then the order of this last system is $\phi(p-1)$ and hence $\phi(p)=p^{2}-\phi(p-1)$ : observing that $\phi(2)=3$, this gives directly $\phi(p)=\frac{1}{2} p(p+1)$. Hence the order of the system is $\frac{1}{2} p(p+1)$.

Suppose $x, y, z$, connected by equations of the form $U=0, V=0, W=0 ; U, V, W$ being linear in $x, y, z$, and homogeneous functions of the orders $m, n, p$ respectively in $\xi, \eta$. By eliminating $x, y, z$, the ratio $\xi: \eta$ will be determined by an equation of the order $m+n+p$; and since when this is known the ratios $x: y: z$ are linearly determinable, we have $m+n+p$ for the order $\mu$ of the system.

Thus, if $m=n=p=2$, selecting the particular system

$$
\begin{aligned}
& a \xi^{2}+2 b \xi \eta+c \eta^{2}=0, \\
& b \xi^{2}+2 c \xi \eta+d \eta^{2}=0, \\
& c \xi^{2}+2 d \xi \eta+e \eta^{2}=0,
\end{aligned}
$$

it is possible in this case to obtain two resulting equations of the orders two and three respectively, and which consequently constitute the system of the sixth order, without containing any extraneous system. In fact, from the identical equation

$$
\begin{array}{r}
e \xi \cdot\left(a \xi^{2}+2 b \xi \eta+c \eta^{2}\right) \\
-(4 d \xi+2 e \eta)\left(b \xi^{2}+2 c \xi \eta+d \eta^{2}\right) \\
+(3 c \xi+2 d \eta)\left(c \xi^{2}+2 d \xi \eta+e \eta^{2}\right) \\
=\left(a e-4 b d+3 c^{2}\right) \xi^{3}
\end{array}
$$


and

$$
\begin{aligned}
& \left(c e-d^{2}\right)\left(a \xi^{2}+2 b \xi \eta+c \eta^{2}\right) \\
+ & (c d-b e)\left(b \xi^{2}+2 c \xi \eta+d \eta^{2}\right) \\
+ & \left(b d-c^{2}\right)\left(c \xi^{2}+2 d \xi \eta+e \eta^{2}\right) \\
& =\left(a c e-a d^{2}-b^{2} e-c^{3}+2 b c d\right) \xi^{2}
\end{aligned}
$$

we deduce

$$
\begin{cases}a e-4 b d+3 c^{2} & =0 \\ a c e-a d^{2}-b^{2} e-c^{3}+2 b c d=0\end{cases}
$$

which form the system in question, and may for shortness be represented by $I=0, J=0$.

The three equations in $\xi, \eta$ may be considered as expressing that

$$
\begin{aligned}
& a \xi^{3}+3 b \xi^{2} \eta+3 c \xi \eta^{2}+d \eta^{3}=0 \\
& b \xi^{3}+3 c \xi^{2} \eta+3 d \xi \eta^{2}+e \eta^{3}=0
\end{aligned}
$$

have a pair of equal roots in common; in other words, that it is possible to satisfy identically

$$
(A \xi+B \eta)\left(a \xi^{3}+3 b \xi^{2} \eta+3 c \xi \eta^{2}+d \eta^{3}\right)+\left(A^{\prime} \xi+B^{\prime} \eta\right)\left(b \xi^{3}+3 c \xi^{2} \eta+3 d \xi \eta^{2}+e \eta^{3}\right)=0 .
$$

Equating to zero the separate terms of this equation, and eliminating $A, B, A^{\prime}, B^{\prime}$, we obtain

$$
\left\|\begin{array}{ccccc}
\cdot & a, & 3 b, & 3 c, & d \\
\cdot & b, & 3 c, & 3 d, & e \\
a, & 3 b, & 3 c, & d, & . \\
b, & 3 c, & 3 d, & e, & .
\end{array}\right\|=0 .
$$

It is not at first sight obvious what connection these equations have with the two, $I=0, J=0$, but by actual expansion they reduce themselves to the following five,

$$
\begin{aligned}
3\left[2\left(c e-d^{2}\right) I-3 e J\right] & =0 \\
3[(b e-c d) I-3 d J] & =0 \\
{\left[-\left(a e+2 b d-3 c^{2}\right) I+9 c J\right] } & =0 \\
3[(a d-b c) I-3 b J] & =0 \\
3\left[2\left(a c-b^{2}\right) I-3 a J\right] & =0
\end{aligned}
$$

which are satisfied by $I=0, J=0$. By the theorem above given, the equations are to be considered as forming a system of the tenth order; the system must therefore be considered as composed of the system $I=0, J=0$, and of a system of the fourth order. The system of the fourth order may be written in the form

$$
\begin{aligned}
& 2\left(a c-b^{2}\right): a d-b c: a e+2 b d-3 c^{2}: b e-c d: 2\left(c e-d^{2}\right): 3 J \\
& =a: b: 3 c: d: \quad e: I \text { : }
\end{aligned}
$$


but to justify this, it must be shown first that these equations reduce themselves to two independent equations; and next that system is really one of the fourth order. We may remark in the first place, that if

$$
u,=a \xi^{4}+4 b \xi^{3} \eta+6 c \xi^{2} \eta^{2}+4 d \xi \eta^{3}+e \eta^{4},
$$

is a perfect square, the coefficients will be proportional to those of $\frac{d^{2} u}{d \xi^{2}} \frac{d^{2} u}{d \eta^{2}}-\left(\frac{d^{2} u}{d \xi d \eta}\right)^{2} \cdot\left(^{1}\right)$ Thus the conditions requisite in order that $u$ may be a perfect square, are given by the system

$$
\begin{gathered}
2\left(a c-b^{2}\right):(a d-b c): a e+2 b d-3 c^{2}: b e-c d: 2\left(c e-d^{2}\right) \\
=\quad a: b: 3 c: d: d e,
\end{gathered}
$$

or these equations are equivalent to two independent equations only (this may be easily verified $\grave{a}$ posteriori); and by writing $3 J$ in the form

$$
e\left(a c-b^{2}\right)-2 d(a d-b c)+c\left(a e+2 b d-3 c^{2}\right)-2 b(b e-c d)+a\left(c e-d^{2}\right),
$$

the remaining equations of the complete system (3) are immediately deduced; thus the latter system contains only two independent equations. (The preceding reasoning shows that the system (3) expresses the conditions in order that the equations

$$
a \xi^{3}+3 b \xi^{2} \eta+3 c \xi \eta^{2}+d \eta^{3}=0, \quad b \xi^{3}+3 c \xi^{2} \eta+3 d \xi \eta^{2}+e \eta^{2}=0,
$$

may have a pair of unequal roots in common: we have already seen that the equations $I=0, J=0$ represent the conditions in order that these two equations may have a pair of equal roots in common.) Finally, to verify $\grave{a}$ posteriori the fact of the system (3) being one only of the fourth order, me may, as Mr Salmon has done in the memoir above referred to, represent the system by the two equations

$$
\begin{aligned}
& a\left(c e-d^{2}\right)-e\left(a c-b^{2}\right)=0, \quad e(a d-b c)-2 b\left(c e-d^{2}\right)=0 \text {, } \\
& \text { that is, by } \quad a d^{2}-e b^{2}=0, \quad 2 b d^{2}-3 b c e+a d e=0 .
\end{aligned}
$$

These equations contain the extraneous system $(a=0, b=0)$ and the extraneous systems $(b=0, d=0)$ and $(d=0, e=0)$, each of which last, as Mr Salmon has remarked from geometrical considerations, counts double, or the system is one of the $4^{\text {th }}$ order only.

${ }^{1}$ More generally whatever be the order of $u$, if $u$ contain a square factor, this square factor may easily be shown to occur in $\frac{d^{2} u}{d \xi^{2}} \frac{d^{2} u}{d \eta^{2}}-\left(\frac{d^{2} u}{d \xi d \eta}\right)^{2}$. 